\title{
Synthesis and In Silico Study of 4-Substituted 1-Aminoanthraquinones
}

\author{
V. I. Shupeniuk ${ }^{a} *$, N. Amaladoss ${ }^{b}$, T. N. $\operatorname{Taras}^{a}$, O. P. Sabadakh ${ }^{a}$, and N. P. Matkivskyi ${ }^{a}$ \\ ${ }^{a}$ Department of the Environment and Chemical Education, Vasyl Stefanyk Precarpathian National University, \\ Ivano-Frankivsk, 76018 Ukraine \\ ${ }^{b}$ Department of Chemistry, Annai College of Arts and Science, Kumbakonam, 612503 India \\ *e-mail:vasyl.shupeniuk@pnu.edu.ua
}

Received January 11, 2021; revised January 22, 2021; accepted January 23, 2021

\begin{abstract}
Eight new 4-substituted 1-amino-9,10-anthraquinones containing a primary amino group were synthesized by nucleophilic substitution of bromine in 1-amino-4-bromo-9,10-anthraquinones. 1-Amino-4-[2-(hydroxyethyl)amino]-9,10-dioxo-9,10-dihydroanthracene-2-sulfonic acid containing a biogenic amine fragment (2-aminoethanol) was converted into the corresponding 1-triazenyl derivatives. The structure of the synthesized compounds was determined on the basis of the LC/MS and ${ }^{13} \mathrm{C}$ and ${ }^{1} \mathrm{H}$ NMR data, and their drug likeness was estimated in silico. Compounds with a good drug likeness score were analyzed by DIGEP-Pred, their possible interactions with proteins were simulated using STRING, and their biological activity was interpreted using the Kyoto Encyclopedia of Genes and Genomes.
\end{abstract}

Keywords: bromaminic acid, Ullmann reaction, LC/MS, 4-substituted 9,10-anthraquinones, triazenes, genes

DOI: $10.1134 / \mathrm{S} 1070428021040126$

INTRODUCTION

Formerly, anthraquinone derivatives were historically important natural dyes. Later, it was found that the planar tricyclic anthraquinone system gives rise to a broad spectrum of biologically important properties (Fig. 1) [1-2]. At present, anthraquinone derivatives are extensively studied as therapeutic agents against COVID-19, specifically acting against 3CLpro and PLpro proteases [3].
A large number of anthraquinone derivatives contain a sulfonate group in the 2-position. These compounds can be synthesized from bromaminic acid sodium salt 1 (Fig. 2) which is the basic starting material for the preparation of biologically active anthraquinone derivatives and numerous dyes [4-6]. In fact, bromaminic acid and its salts are widely used as intermediate products for the synthesis of anthraquinone derivatives, including acid dyes, via substitution of the 4-bromine atom by (aryl)alkylamino group [7-9].

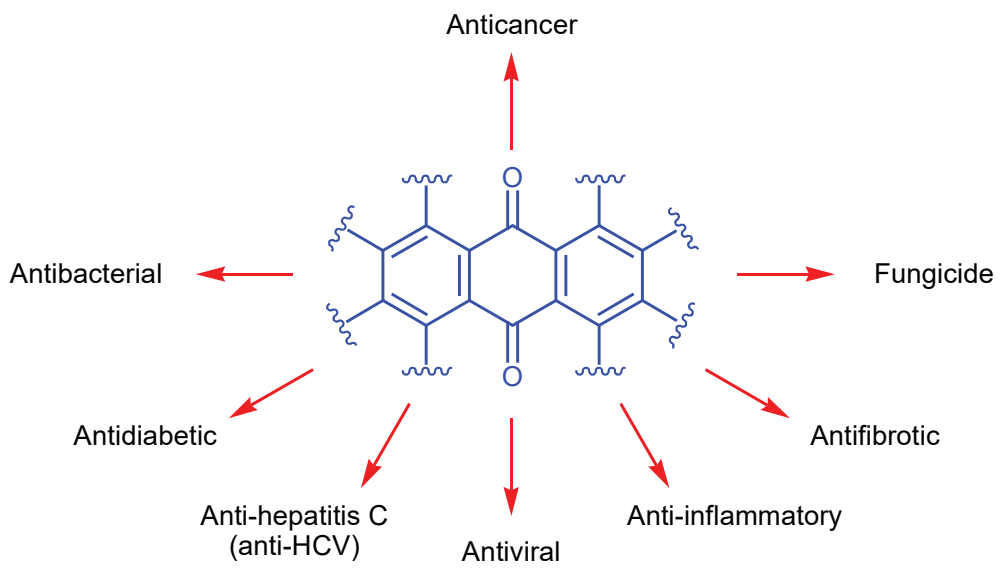

Fig. 1. Biological activity of anthraquinone derivatives. 


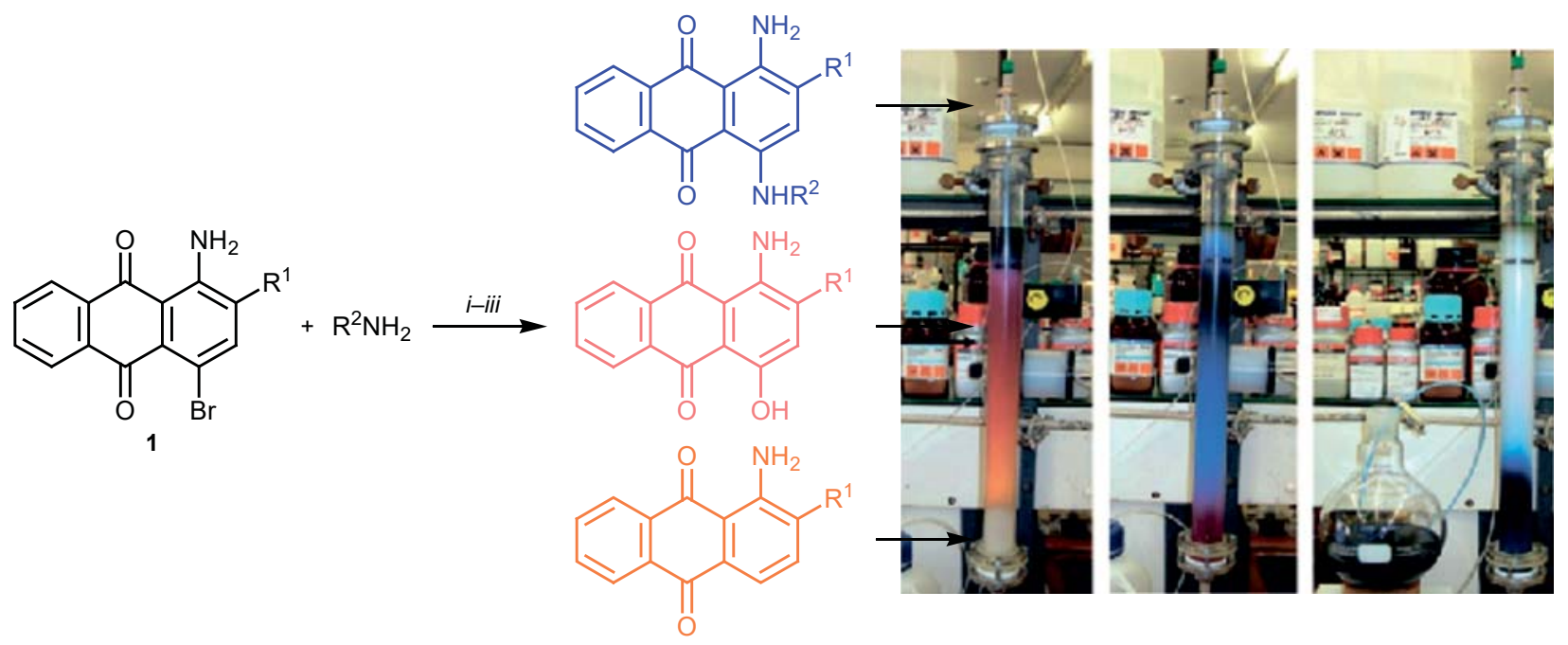

$\mathrm{R}^{1}=\mathrm{SO}_{3} \mathrm{Na}, \mathrm{R}^{2}=\mathrm{Alk}, \mathrm{Ar} ; i: \mathrm{CuCl}, \mathrm{Na}_{2} \mathrm{CO}_{3}, \mathrm{Na}_{2} \mathrm{SO}_{3}, \mathrm{H}_{2} \mathrm{O}$, r.t., 8-24 h or $120^{\circ} \mathrm{C}, 8-10$ h; $i \mathrm{i}: \mathrm{CuSO}_{4}, \mathrm{Na}_{2} \mathrm{CO}_{3}, \mathrm{H}_{2} \mathrm{O}, 120^{\circ} \mathrm{C}, 12-48$ h; iii: phosphate buffer, $\mathrm{pH} 6-7, \mathrm{Cu}(0)$, microwave irradiation, $100-120^{\circ} \mathrm{C}, 5-24 \mathrm{~min}$.

Fig. 2. Synthesis of 4-substituted 1-aminoanthraquinones.

There are almost no published data on chemical properties of triazenyl-substituted anthraquinones $[10,11]$. Triazene moiety is a known alkylating carcinolytic group. It was introduced into anthraquinone molecule by azo coupling of anthraquinone-1-diazonium salt with various aliphatic and aromatic amines.

\section{RESULTS AND DISCUSSION}

While developing an optimal procedure for the substitution of bromine in bromaminic acid 1, we tried different known nucleophilic substitution methods $[12,13]$ and performed a series of reactions of bromaminic acid $\mathbf{1}$ and its 2-methyl analog 2 with 2-aminoethanol under different conditions; however, the yields were not always satisfactory (Scheme 1). We found that the most efficient procedure was to react compound 1 or $\mathbf{2}$ with 2-aminoethanol in aqueous medium in the presence of a mixture of copper(II) and iron(II) salts [14]. In this case, the yield of target 1-amino-4[(2-hydroxyethyl)amino]-9,10-dioxo-9,10-dihydroan- thracene-2-sulfonic acid (5) was 96\% $(\mathrm{m} / \mathrm{z} 362.0$ $\left.[M+\mathrm{H}]^{+}\right)$. The structure of 5 was confirmed by ${ }^{1} \mathrm{H}$ and ${ }^{13} \mathrm{C}$ NMR spectra and elemental analysis. Compound 6 was obtained in $65 \%$ yield, and its ${ }^{1} \mathrm{H}$ NMR spectrum showed signals of the ethylene moiety at $\delta 3.50$ and $3.67 \mathrm{ppm}$. Under these conditions, broaminic acid 1 was reacted with other primary aliphatic amines to obtain 1,4-diaminoanthraquinone derivatives 7-12 (Scheme 2).

Almost all these reactions were accompanied by side formation of 1-amino-4-hydroxy-9,10-dioxo-9,10dihydroanthracene-2-sulfonic acid (4) due to concurrent attack of hydroxy nucleophile (Table 1). Furthermore, there was a clear relation between the $\mathrm{p} K_{\mathrm{a}}$ value of the amine and the purity of the product (Scheme 2).

Synthesis of triazenes. In the next step of our study, aminoanthraquinone $\mathbf{5}$ was subjected to diazotization with sodium nitrite in aqueous medium in the presence of $\mathrm{HCl}$ at $0-5^{\circ} \mathrm{C}$ [15-16] (Scheme 3). The subsequent azo coupling of diazonium salts $\mathbf{1 3}$ with secondary and primary amines afforded triazenes $\mathbf{1 4}$

\section{Scheme 1.}<smiles>[R]c1cc([R])c2c(c1N)C(=O)c1ccccc1C2=O</smiles>

1, $\mathrm{R}^{1}=\mathrm{SO}_{3} \mathrm{H} ; \mathbf{2}, \mathrm{R}^{1}=\mathrm{Me} ; \mathbf{3}, \mathrm{R}^{1}=\mathrm{SO}_{3} \mathrm{H}, \mathrm{R}^{2}=\mathrm{H} ; \mathbf{4}, \mathrm{R}^{1}=\mathrm{SO}_{3} \mathrm{H}, \mathrm{R}^{2}=\mathrm{OH}$

5, $\mathrm{R}^{1}=\mathrm{SO}_{3} \mathrm{H}, \mathrm{R}^{2}=\mathrm{NHCH}_{2} \mathrm{CH}_{2} \mathrm{OH} ; \mathbf{6}, \mathrm{R}^{1}=\mathrm{Me}, \mathrm{R}^{2}=\mathrm{NHCH}_{2} \mathrm{CH}_{2} \mathrm{OH}$. 
Scheme 2.<smiles>[R2]Nc1cc(S(=O)(=O)O)c(N)c2c1C(=O)c1ccccc1C2=O</smiles>

7, $\mathrm{R}=$ cyclo $-\mathrm{C}_{5} \mathrm{H}_{9}\left(65 \%, \mathrm{p} K_{\mathrm{a}}=10.5\right) ; 8, \mathrm{R}=i-\operatorname{Pr}\left(68 \%, \mathrm{p} K_{\mathrm{a}}=10.6\right) ; 9, \mathrm{R}=$ furan-2-ylmethyl $\left(84 \%, \mathrm{p} K_{\mathrm{a}}=10.62\right) ;$ 10, $\mathrm{R}=$ morpholin-4-ylamino $\left(85 \%, \mathrm{p} K_{\mathrm{a}}=10.67\right) ; 11, \mathrm{R}=\operatorname{Pr}\left(98 \%, \mathrm{p} K_{\mathrm{a}}=10.71\right) ; 12, \mathrm{R}=\mathrm{Bu}\left(100 \%, \mathrm{p} K_{\mathrm{a}}=10.77\right)$.

Scheme 3.<smiles>Nc1c(N(CO)CO)cc(S(=O)(=O)O)c2c1C(=O)c1ccccc1C2=O</smiles>

$\mathrm{NaNO}_{2}, \mathrm{HCl}, 0-5^{\circ} \mathrm{C}$<smiles>[NH2+]c1c(S(=O)(=O)[O-])cc(NCO)c2c1C(=O)c1ccccc1C2=O</smiles>

Scheme 4.<smiles>Nc1cc([Se])c([N+](=O)[O-])c2c1C(=O)c1ccccc1C2=O</smiles>

$\mathrm{R}^{1} \mathrm{R}^{2} \mathrm{NH}, \mathrm{Na}_{2} \mathrm{CO}_{3}$<smiles>[R17]N=Nc1c(O)cc(N=[Z17])c2c1C(=O)c1ccccc1C2=O</smiles>

14, $\mathrm{R}^{1}=\mathrm{R}^{2}=\mathrm{CH}_{2} \mathrm{CH}_{2} \mathrm{OH} ; 15, \mathrm{R}^{1} \mathrm{R}^{2} \mathrm{~N}=$ morpholin-4-yl; 16, $\mathrm{R}^{1}=\mathrm{R}^{2}=\mathrm{Et} ; 1$ 17, $\mathrm{R}^{1}=\mathrm{H}, \mathrm{R}^{2}=4-\mathrm{HOC}(\mathrm{O}) \mathrm{C}_{6} \mathrm{H}_{4}$.

17 (Scheme 4). This reaction was not always smooth, depending on the properties of the initial amine and stability of the diazo coupling products. The structure of 14-17 was confirmed by spectral data. The ${ }^{1} \mathrm{H}$ NMR spectra of 14-17 showed aromatic protons signals in the region $\delta 7.50-8.70 \mathrm{ppm}$.

Biological activity and drug likeness. Among the 12 synthesized compounds, we identified triazene derivatives with good drug likeness scores. In particular, 4-aminobenzoic acid derivative 17 (M 510.08) showed the highest drug likeness score $(+0.06)$. Table 2 contains the detailed drug likeness parameters of the synthesized compounds.

Triazene 14 modulated the largest number of genes (10). The effect on the CHEK1 gene responsible for the p53 signaling pathway directly involved in immune strengthening was revealed. Furthermore, the gene set enrichment analysis showed modulation of 15 different biological pathways, among which cancer pathways are modulated mainly by regulation of three genes (SP1, IL23A, NFE2L2). The results of gene set enrichment analysis of protein modulation by anthraquinone

Table 1. Synthesis of compounds 7-12

\begin{tabular}{c|c|c}
\hline Compound no. & Yield, \% & Impurity of $\mathbf{4 ,} \%$ \\
\hline $\mathbf{7}$ & 65 & 25 \\
$\mathbf{8}$ & 68 & 20 \\
$\mathbf{9}$ & 84 & 15 \\
$\mathbf{1 0}$ & 85 & 5 \\
$\mathbf{1 1}$ & 98 & 1 \\
$\mathbf{1 2}$ & 100 & - \\
\hline
\end{tabular}


Table 2. Drug likeness parameters of compounds $\mathbf{5}-\mathbf{1} 7^{\mathrm{a}}$

\begin{tabular}{c|c|c|c|c|c|c|c|c}
\hline $\begin{array}{c}\text { Compd. } \\
\text { no. }\end{array}$ & Formula & Molecular weight & NHBA & NHBD & Log $P$ & TPSA, $\AA^{2}$ & $V, \AA^{3}$ & DLS \\
\hline $\mathbf{5}$ & $\mathrm{C}_{16} \mathrm{H}_{14} \mathrm{~N}_{2} \mathrm{O}_{6} \mathrm{~S}$ & 362.06 & 6 & 5 & -0.57 & 114.29 & 307.29 & -0.73 \\
$\mathbf{6}$ & $\mathrm{C}_{17} \mathrm{H}_{16} \mathrm{~N}_{2} \mathrm{O}_{3}$ & 296.12 & 3 & 4 & 2.76 & 71.87 & 288.83 & -0.16 \\
$\mathbf{7}$ & $\mathrm{C}_{19} \mathrm{H}_{18} \mathrm{~N}_{2} \mathrm{O}_{5} \mathrm{~S}$ & 386.09 & 5 & 5 & 1.40 & 97.61 & 340.44 & -0.19 \\
$\mathbf{8}$ & $\mathrm{C}_{17} \mathrm{H}_{16} \mathrm{~N}_{2} \mathrm{O}_{5} \mathrm{~S}$ & 360.08 & 5 & 4 & 0.72 & 96.76 & 317.25 & -0.66 \\
$\mathbf{9}$ & $\mathrm{C}_{19} \mathrm{H}_{18} \mathrm{~N}_{2} \mathrm{O}_{6} \mathrm{~S}$ & 402.09 & 6 & 4 & 0.24 & 106.48 & 355.56 & -0.17 \\
$\mathbf{1 0}$ & $\mathrm{C}_{18} \mathrm{H}_{17} \mathrm{~N}_{3} \mathrm{O}_{6} \mathrm{~S}$ & 403.08 & 7 & 4 & -0.53 & 110.07 & 353.50 & -0.27 \\
$\mathbf{1 1}$ & $\mathrm{C}_{17} \mathrm{H}_{16} \mathrm{~N}_{2} \mathrm{O}_{5} \mathrm{~S}$ & 360.08 & 5 & 4 & 0.82 & 97.63 & 317.22 & -0.57 \\
$\mathbf{1 2}$ & $\mathrm{C}_{18} \mathrm{H}_{18} \mathrm{~N}_{2} \mathrm{O}_{5} \mathrm{~S}$ & 374.09 & 5 & 4 & 1.34 & 97.63 & 335.12 & -0.69 \\
$\mathbf{1 4}$ & $\mathrm{C}_{18} \mathrm{H}_{18} \mathrm{~N}_{4} \mathrm{O}_{8} \mathrm{~S}$ & 450.08 & 10 & 5 & -0.74 & 150.24 & 380.77 & -0.79 \\
$\mathbf{1 5}$ & $\mathrm{C}_{20} \mathrm{H}_{22} \mathrm{~N}_{4} \mathrm{O}_{6} \mathrm{~S}$ & 446.13 & 8 & 3 & 1.18 & 117.64 & 399.55 & -0.60 \\
$\mathbf{1 6}$ & $\mathrm{C}_{20} \mathrm{H}_{20} \mathrm{~N}_{4} \mathrm{O}_{7} \mathrm{~S}$ & 460.11 & 9 & 3 & 0.04 & 126.10 & 402.62 & -0.23 \\
$\mathbf{1 7}$ & $\mathrm{C}_{23} \mathrm{H}_{18} \mathrm{~N}_{4} \mathrm{O}_{8} \mathrm{~S}$ & 510.08 & 10 & 5 & 1.01 & 155.40 & 434.50 & 0.06 \\
\hline
\end{tabular}

${ }^{\text {a }}$ NHBA is the number of hydrogen bond acceptor centers, NHBD is the number of hydrogen bond donor centers, Log $P$ is the lipophilicity coefficient, TPSA is the topological polar surface area, $V$ is the molecular volume, and DLS is the drug likeness score.

Table 3. Analysis of proteins modulated by anthraquinone derivatives

\begin{tabular}{c|l|c|l}
\hline Pathway ID & \multicolumn{1}{|c|}{ Description } & Number of genes & \multicolumn{1}{|c}{ Matching genes } \\
\hline hsa05200 & Pathways in cancer & 3 & SP1, IL23A, NFE2L2 \\
hsa05164 & Influenza A & 2 & NXT2, SP1 \\
hsa05133 & Pertussis & 2 & SP1, IL23A \\
ko04625 & C-Type lectin receptor signaling pathway & 2 & SP1, IL23A \\
hsa05166 & HTLV-I infection & 1 & CHEK1 \\
hsa05014 & Amyotrophic lateral sclerosis (ALS) & 1 & SP1 \\
hsa05152 & Tuberculosis & 1 & IL23A \\
hsa05224 & Breast cancer & 1 & SP1 \\
hsa05204 & Chemical carcinogenesis & 1 & CBR1 \\
hsa05225 & Hepatocellular carcinoma & 1 & NFE2L2 \\
hsa05203 & Viral carcinogenesis & 1 & CHEK1 \\
hsa04216 & Ferroptosis & 1 & GSS \\
hsa05323 & Rheumatoid arthritis & 1 & IL23A \\
hsa01524 & Platinum drug resistance & 1 & TOP2A \\
hsa04115 & p53 signaling pathway & 1 & CHEK1 \\
\hline
\end{tabular}

RUSSIAN JOURNAL OF ORGANIC CHEMISTRY Vol. 57 No. 42021 


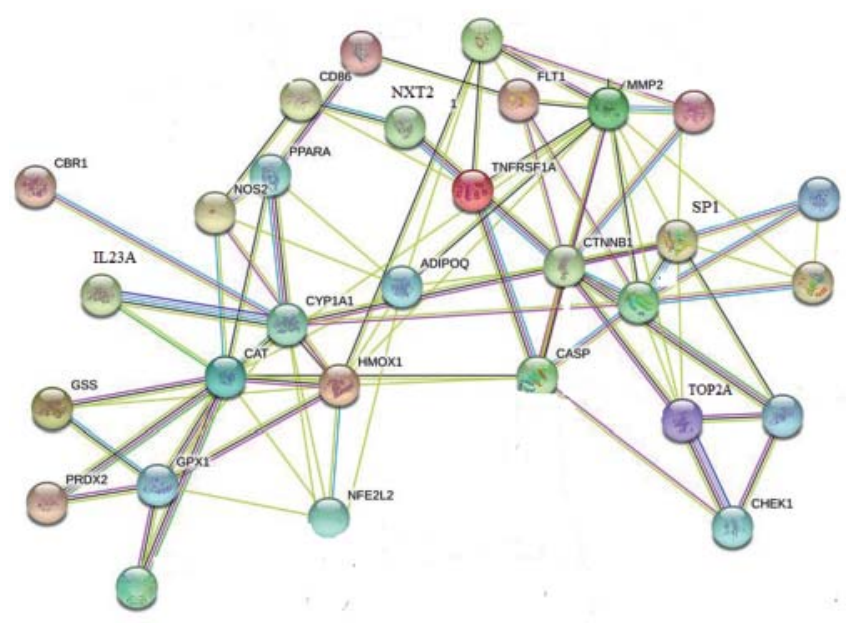

Fig. 3. Interactions between regulated proteins.

derivatives and the corresponding gene codes are collected in Table 3. Figure 3 shows interactions between modulated proteins.

\section{EXPERIMENTAL}

All chemicals were obtained from commercial sources and were used without further purification. The melting points were measured in open capillary tubes. The ${ }^{1} \mathrm{H}$ NMR spectra were recorded on a Varian 400 spectrometer at $400 \mathrm{MHz}$ using DMSO- $d_{6}$ as solvent unless otherwise stated. The mass spectra were run on an Agilent 1100 Series high-performance liquid chromatograph equipped with a diode array detector and an Agilent mass-selective detector with the possibility of rapidly switching between positive and negative ionization modes. The progress of reactions was monitored by TLC on DC-Fertigfolien Alugram Xtra Sil G/UV254 silica gel plates (Germany).

Anthraquinone derivatives with good drug likeness scores were tested by DIGEP-Pred [17] to identify target proteins (upregulated and downregulated proteins) with a probable activity of 0.4 . The list of regulated proteins was loaded to STRING [18] to identify biological process, cellular function, and combined gene set. In addition, possible modulation pathways were identified using the Kyoto Encyclopedia of Genes and Genomes.

General procedure for the synthesis of anthraquinone derivatives 5-12. Bromaminic acid $\mathbf{1}$ (4.04 g, $0.01 \mathrm{~mol})$ was dissolved in $40 \mathrm{~mL}$ of hot water $\left(70-80^{\circ} \mathrm{C}\right)$, the corresponding amine $(0.015 \mathrm{~mol})$ and sodium hydrogen carbonate $(0.02 \mathrm{~mol})$ were added in succession, and copper(II) sulfate (0.05 g) and iron(II) sulfate $(0.05 \mathrm{~g})$ were then added. The mixture was stirred, heated to $90^{\circ} \mathrm{C}$, and kept at that temperature for $4 \mathrm{~h}$. The progress of the reaction was monitored by the disappearance of bromaminic acid 1 (o-xyleneacetone, 4:6). After completion of the reaction, the mixture was cooled to room temperature and acidified with concentrated aqueous $\mathrm{HCl}$, and the precipitate was filtered off and washed with $20 \%$ aqueous sodium chloride $(60 \mathrm{~mL})$. The blue moist product was dissolved in hot water $(50 \mathrm{~mL})$ and precipitated with concentrated aqueous $\mathrm{HCl}(3 \mathrm{~mL})$.

1-Amino-4-[(2-hydroxyethyl)amino]-9,10-dioxo9,10-dihydroanthracene-2-sulfonic acid (5). Yield $96 \%$, blue solid, mp $287-289^{\circ} \mathrm{C} .{ }^{1} \mathrm{H}$ NMR spectrum, $\delta$, ppm: $3.49 \mathrm{~d}\left(2 \mathrm{H}, \mathrm{CH}_{2}\right), 3.69 \mathrm{~d}\left(2 \mathrm{H}, \mathrm{CH}_{2}\right), 7.73 \mathrm{~s}(1 \mathrm{H}$, $3-\mathrm{H}), 7.85 \mathrm{t}\left(2 \mathrm{H}, \mathrm{H}_{\text {arom }}, J=7.7 \mathrm{~Hz}\right), 8.25 \mathrm{~d}\left(2 \mathrm{H}, \mathrm{H}_{\text {arom }}\right.$, $J=8.0 \mathrm{~Hz}) .{ }^{13} \mathrm{C}$ NMR spectrum, $\delta_{\mathrm{C}}$, ppm: $45.29\left(\mathrm{CH}_{2}\right)$, $60.24\left(\mathrm{CH}_{2} \mathrm{OH}\right), 109.38,109.67,121.16,126.19$, 126.36, 132.88, 133.03, 134.43, 134.47, 143.5, 143.79, $145.84\left(\mathrm{C}_{\text {arom }}\right), 181.17,182.12(\mathrm{C}=\mathrm{O})$. Mass spectrum: $\mathrm{m} / \mathrm{z} 364.0[M+\mathrm{H}]^{+}$. Found, \%: C 53.10; H 4.40; $\mathrm{N}$ 6.90; $\mathrm{S} 7.80 . \mathrm{C}_{18} \mathrm{H}_{18} \mathrm{~N}_{2} \mathrm{O}_{7} \mathrm{~S}$. Calculated, \%: C 53.07; $\mathrm{H}$ 4.42; N 6.87; S 7.86. M 364 .

1-Amino-4-[(2-hydroxyethyl)amino]-2-methylantracene-9,10-dione (6). Yield 59\%, blue solid, mp $300^{\circ} \mathrm{C}$. ${ }^{1} \mathrm{H}$ NMR spectrum, $\delta$, ppm $2.30 \mathrm{~s}(3 \mathrm{H}$, $\left.\mathrm{CH}_{3}\right), 3.50-3.60 \mathrm{~m}\left(2 \mathrm{H}, \mathrm{CH}_{2}\right), 3.67 \mathrm{~d}\left(2 \mathrm{H}, \mathrm{CH}_{2}, J=\right.$ $5.2 \mathrm{~Hz}), 7.34 \mathrm{~s}(1 \mathrm{H}, 3-\mathrm{H}), 7.68-7.86 \mathrm{~m}\left(4 \mathrm{H}, \mathrm{H}_{\text {arom }}\right)$. Mass spectrum: $m / z 297.0[M+\mathrm{H}]^{+} . \mathrm{C}_{17} \mathrm{H}_{17} \mathrm{~N}_{2} \mathrm{O}_{3}$. M 297.

1-Amino-4-[(propan-2-yl)amino]-9,10-dioxo9,10-dihydroanthracene-2-sulfonic acid (8). Yield $68 \%$, blue solid, mp $260-262{ }^{\circ} \mathrm{C} .{ }^{1} \mathrm{H}$ NMR spectrum, $\delta$, ppm: $1.10 \mathrm{~s}\left(3 \mathrm{H}, \mathrm{CH}_{3}\right), 2.20-2.25 \mathrm{~m}\left(6 \mathrm{H}, \mathrm{CH}_{2}\right), 7.70 \mathrm{t}$ $\left(3 \mathrm{H}, \mathrm{H}_{\text {arom }}\right), 8.00 \mathrm{~s}\left(2 \mathrm{H}, \mathrm{H}_{\text {arom }}\right), 10.50 \mathrm{~s}(1 \mathrm{H}, \mathrm{NH})$. Mass spectrum: $m / z 361.2[M+\mathrm{H}]^{+}$. $\mathrm{C}_{17} \mathrm{H}_{16} \mathrm{~N}_{2} \mathrm{O}_{5} \mathrm{~S} . M 361$.

1-Amino-4-[(morpholin-4-yl)amino]-9,10-dioxo9,10-dihydroanthracene-2-sulfonic acid (10). Yield $85 \%$, blue solid, mp $282^{\circ} \mathrm{C} .{ }^{1} \mathrm{H}$ NMR spectrum, $\delta$, ppm: $2.00-2.10 \mathrm{~m}\left(4 \mathrm{H}, \mathrm{CH}_{2}\right), 3.30-4.00 \mathrm{~m}\left(4 \mathrm{H}, \mathrm{CH}_{2}\right)$, $7.70 \mathrm{~s}\left(3 \mathrm{H}, \mathrm{H}_{\text {arom }}\right), 8.20 \mathrm{~s}\left(3 \mathrm{H}, \mathrm{H}_{\text {arom }}, \mathrm{NH}\right)$. Mass spectrum: $m / z 406[M+\mathrm{H}]^{+} . \mathrm{C}_{18} \mathrm{H}_{17} \mathrm{~N}_{3} \mathrm{O}_{6} \mathrm{~S} . M 402$.

1-Amino-4-(propylamino)-9,10-dioxo-9,10dihydroanthracene-2-sulfonic acid (11). Yield 98\%, blue solid, mp $262{ }^{\circ} \mathrm{C}$. ${ }^{1} \mathrm{H}$ NMR spectrum, $\delta$, ppm: $1.00 \mathrm{~s}\left(3 \mathrm{H}, \mathrm{CH}_{3}\right), 1.30-1.35 \mathrm{~m}\left(6 \mathrm{H}, \mathrm{CH}_{2}\right), 7.80 \mathrm{t}(3 \mathrm{H}$, $\left.\mathrm{H}_{\text {arom }}\right), 8.20 \mathrm{~s}\left(2 \mathrm{H}, \mathrm{H}_{\text {arom }}\right), 10.80 \mathrm{~s}(1 \mathrm{H}, \mathrm{NH})$. Mass spectrum: $m / z 362.2[M+\mathrm{H}]^{+} . \mathrm{C}_{17} \mathrm{H}_{16} \mathrm{~N}_{2} \mathrm{O}_{5} \mathrm{~S} . M 361$. 
1-Amino-4-(butylamino)-9,10-dioxo-9,10-dihydroanthracene-2-sulfonic acid (12). Yield 100\%, blue solid, mp $290-292^{\circ} \mathrm{C} .{ }^{1} \mathrm{H}$ NMR spectrum, $\delta$, ppm: $1.00 \mathrm{~s}\left(3 \mathrm{H}, \mathrm{CH}_{3}\right), 1.60 \mathrm{~s}\left(2 \mathrm{H}, \mathrm{CH}_{2}\right), 7.80 \mathrm{~s}\left(3 \mathrm{H}, \mathrm{H}_{\text {arom }}\right)$, $8.20 \mathrm{~s}\left(2 \mathrm{H}, \mathrm{H}_{\text {arom }}\right), 10.70 \mathrm{~s}(1 \mathrm{H}, \mathrm{NH})$. Mass spectrum: $m / z: 375.5[M+\mathrm{H}]^{+} . \mathrm{C}_{18} \mathrm{H}_{18} \mathrm{~N}_{2} \mathrm{O}_{5} \mathrm{~S} . M 375$.

1-(Diazyn-1-ium-l-yl)-4-[(2-hydroxyethyl)amino]-9,10-dioxo-9,10-dihydroanthracene-2-sulfonic acid (13). A 50-mL round-bottom flask equipped with a magnetic stirrer was charged with a solution of anthraquinone $5(0.1 \mathrm{mmol})$ in $1 \mathrm{M}$ aqueous $\mathrm{HCl}$ $(5.0 \mathrm{~mL})$. The solution was cooled to $0-5^{\circ} \mathrm{C}$ in an ice bath, a solution of sodium nitrite $(0.2 \mathrm{mmol})$ in $0.5 \mathrm{~mL}$ of distilled water was added dropwise, maintaining the temperature at $0-5^{\circ} \mathrm{C}$, and the mixture was stirred for $5 \mathrm{~min}$ at that temperature.

Triazene derivatives 14-17 (general procedure). The mixture containing diazonium salt $\mathbf{1 3}$ was allowed to warm up to room temperature, a solution of the corresponding amine $(0.15 \mathrm{mmol})$ in $5 \mathrm{~mL}$ of ethanol was added, and the mixture was stirred for $\sim 30 \mathrm{~s}$ at room temperature. The progress of the reaction was monitored by change of the color of the reaction mixture (the color changed from blue to red after diazotization, and the final product was purple), as well as by RP-TLC using acetone-water $(2: 3)$ as eluent. The product was purified by reversed phase column chromatography (RP-18) using water as eluent.

1-[3,3-Bis(2-hydroxyethyl)triaz-1-en-1-yl]-4-[(2hydroxyethyl)amino]-9,10-dioxo-9,10-dihydroanthracene-2-sulfonic acid (14). Yield 80\%. ${ }^{1} \mathrm{H}$ NMR spectrum, $\delta$, ppm: $2.99 \mathrm{~s}\left(4 \mathrm{H}, \mathrm{CH}_{2}\right), 3.40 \mathrm{~d}\left(4 \mathrm{H}, \mathrm{CH}_{2}\right.$, $J=11.2 \mathrm{~Hz}), 3.65 \mathrm{~s}\left(4 \mathrm{H}, \mathrm{CH}_{2}\right), 5.08 \mathrm{~s}(\mathrm{OH}), 5.25 \mathrm{~s}$ $(2 \mathrm{H}, \mathrm{OH}), 7.85 \mathrm{~s}\left(2 \mathrm{H}, \mathrm{H}_{\text {arom }}\right), 8.15-8.20 \mathrm{~m}\left(2 \mathrm{H}, \mathrm{H}_{\text {arom }}\right)$, $8.69 \mathrm{~s}\left(2 \mathrm{H}, \mathrm{H}_{\text {arom }}\right), 9.87 \mathrm{~s}(1 \mathrm{H}, \mathrm{NH})$. Mass spectrum: $m / z 360.9[M+\mathrm{H}]^{+} . \mathrm{C}_{16} \mathrm{H}_{12} \mathrm{~N}_{2} \mathrm{O}_{6} \mathrm{~S} . M 361$.

4-[(2-Hydroxyethyl)amino]-1-[(E)-(morpholin-4yl)diazenyl]-9,10-dioxo-9,10-dihydroanthracene-2sulfonic acid (15). Yield 90\%. mp $>300{ }^{\circ} \mathrm{C} .{ }^{1} \mathrm{H}$ NMR spectrum, $\delta$, ppm: in DMSO- $d_{6}: 3.37 \mathrm{t}\left(1 \mathrm{H}, \mathrm{CH}_{2}\right.$, morpholine), $3.43 \mathrm{t}\left(2 \mathrm{H}, \mathrm{CH}_{2}\right.$, morpholine), 3.70 $3.75 \mathrm{~m}\left(3 \mathrm{H}, \mathrm{CH}_{2}\right.$, morpholine $), 4.95 \mathrm{~s}\left(5 \mathrm{H}, \mathrm{CH}_{2}\right)$, $7.62 \mathrm{~s}(1 \mathrm{H}, 3-\mathrm{H}), 7.86 \mathrm{~s}\left(1 \mathrm{H}, \mathrm{H}_{\text {arom }}\right), 7.92 \mathrm{t}\left(1 \mathrm{H}, \mathrm{H}_{\text {arom }}\right.$, $J=8.0 \mathrm{~Hz}), 8.17 \mathrm{~d}\left(1 \mathrm{H}, \mathrm{H}_{\text {arom }}, J=6.4 \mathrm{~Hz}\right), 8.23 \mathrm{~d}(1 \mathrm{H}$, $\left.\mathrm{H}_{\text {arom }}, J=7.6 \mathrm{~Hz}\right), 9.89 \mathrm{~s}(1 \mathrm{H}, \mathrm{OH})$; in DMSO- $d_{6}$ $\mathrm{CCl}_{4}: 3.46 \mathrm{~s}\left(2 \mathrm{H}, \mathrm{CH}_{2}\right), 3.73 \mathrm{~s}\left(1 \mathrm{H}, \mathrm{CH}_{2}\right), 7.67 \mathrm{~d}(1 \mathrm{H}$, $3-\mathrm{H}, J=6.8 \mathrm{~Hz}), 7.87-7.90 \mathrm{~m}\left(2 \mathrm{H}, \mathrm{H}_{\text {arom }}\right), 8.18 \mathrm{t}(1 \mathrm{H}$, $\left.\mathrm{H}_{\text {arom }}, J=6.0 \mathrm{~Hz}\right), 8.28 \mathrm{~d}\left(1 \mathrm{H}, \mathrm{H}_{\text {arom }}, J=4.8 \mathrm{~Hz}\right)$, $9.90 \mathrm{~s}(1 \mathrm{H}, \mathrm{OH})$. Mass spectrum: $m / z 460[M]^{+}$.
1-(3,3-Diethyltriaz-1-en-1-yl)-4-[(2-hydroxyethyl)amino]-9,10-dioxo-9,10-dihydroanthracene-2sulfonic acid (16). Yield 52\%. ${ }^{1} \mathrm{H}$ NMR spectrum, $\delta$, ppm: $1.18 \mathrm{~s}\left(3 \mathrm{H}, \mathrm{CH}_{3}\right), 2.86 \mathrm{~d}\left(3 \mathrm{H}, \mathrm{CH}_{3}, J=4.8 \mathrm{~Hz}\right)$, $3.40 \mathrm{~s}\left(2 \mathrm{H}, \mathrm{CH}_{2}\right), 7.66-7.70 \mathrm{~m}(1 \mathrm{H}, 3-\mathrm{H}), 8.04-8.30 \mathrm{~m}$ $\left(8 \mathrm{H}, \mathrm{H}_{\text {arom }}\right), 9.01 \mathrm{~s}(1 \mathrm{H}, \mathrm{OH})$. Mass spectrum: $\mathrm{m} / \mathrm{z} 425.9[\mathrm{M}]^{+}$.

4-(3-\{4-[(2-Hyd roxyethyl)amino]-2-sulfo-9,10dioxo-9,10-dihydroanthracen-1-yl \}triaz-2-en-1-yl)benzoic acid (17). Yield $95 \%, \mathrm{mp}>300^{\circ} \mathrm{C} .{ }^{1} \mathrm{H}$ NMR spectrum, $\delta$, ppm: $1.10 \mathrm{~s}\left(1 \mathrm{H}, \mathrm{CH}_{2}\right), 3.46-3.50 \mathrm{~m}(2 \mathrm{H}$, $\left.\mathrm{CH}_{2}, J=16.0 \mathrm{~Hz}\right), 7.20 \mathrm{~d}\left(8 \mathrm{H}, \mathrm{H}_{\text {arom }}, J=7.6 \mathrm{~Hz}\right)$, $7.80 \mathrm{~d}\left(3 \mathrm{H}, \mathrm{H}_{\text {arom }}, J=7.6 \mathrm{~Hz}\right), 7.90 \mathrm{~s}(4 \mathrm{H}, \mathrm{NH}, \mathrm{OH})$. Mass spectrum: $m / z 466\left[M-\mathrm{C}_{22} \mathrm{H}_{17} \mathrm{O}_{6} \mathrm{CH}_{4}\right]^{+}$.

\section{CONCLUSIONS}

The results of computer simulation suggest the possibility of therapeutic effect of the synthesized substituted anthraquinone derivatives, which needs to be verified using experimental protocols.

\section{FUNDING}

This study was performed under financial support by the Ministry of Education and Science of Ukraine (project no. 0119U103131).

\section{CONFLICT OF INTEREST}

The authors declare the absence of conflict of interest.

\section{SUPPLEMENTARY INFORMATION}

The online version contains supplementary material available at https://doi.org/10.1134/S1070428021040126.

\section{REFERENCES}

1. Hussain, H., Al-Harrasi, A., Al-Rawahi, A., Green, I., Csuk, R., Ahmed, I., Shan, A., Abbas, G., Rehman, N., and Ullah, R., Expert Opin. Ther. Pat., 2015, vol. 25, p. 1053. https://doi.org/10.1517/13543776.2015.1050793

2. Malik, E. and Muller, C., Med. Res. Rev., 2016, vol. 36, p. 705.

https://doi.org/10.1002/med.21391

3. Khanal, P., Patil, B.M., Chand, J., and Naaz, Y., Nat. Prod. Bioprospect., 2020, vol. 10, p. 325. https://doi.org/10.1007/s13659-020-00260-2

4. Baqi, Y. and Muller, C.E., Org. Lett., 2007, vol. 9, p. 1271.

https://doi.org/10.1021/ol070102v 
5. Baqi, Y. and Muller, C.E., Nat. Protoc., 2010, vol. 5, p. 945 . https://doi.org/10.1038/nprot.2010.63

6. Baqi, Y., Alzeler, K., Koze, M., and Muller, C.E., J. Med. Chem., 2009, vol. 52, p. 3784. https://doi.org/10.1021/jm9003297

7. Malik, E.M., Baqi, Y., and Müller, C.E., Beilstein J. Org. Chem., 2015, vol. 11, p. 2326. https://doi.org/10.3762/bioc.11.253

8. Roy, S., Large, J.R., Akande, A.M., Kshatri, A., Webb, T.I., Domene, C., Sergeant, G.P., Mchale, N.G., Thornbury, K.D., and Hollywood, M.A., Eur. J. Med. Chem., 2004, vol. 75, p. 426.

https://doi.org/10.1016/j.ejmech.2014.01.035

9. Fiene, A., Baqi, Y., Malik, E.M., Newton, P., Li, W., Lee, S.-Y., Hartland, L.E., and Muller, C.E., Bioorg. Med. Chem., 2016, vol. 24, p. 4363. https://doi.org/10.1016/j.bmc.2016.07.027

10. Sabadakh, O.P., Taras, T.N., Luchkevich, E.R., and Novikov, V.P., Russ. J. Org. Chem., 2015, vol. 51, p. 277. https://doi.org/10.1134/S1070428015020244

11. Bulgakova, N.A. and Gornostaev, L.M., J. Org. Chem., 2001, vol. 37, p. 1351. https://doi.org/10.1023/A:1013164528653
12. Topanov, A.P., Mashevskaya, I.V., Dmitriev, M.V., and Maslivets, A.N., Russ. J. Org. Chem., 2020, vol. 56, p. 719. https://doi.org/10.1134/S1070428020040247

13. Kaur, G., Utreja, D., Jain, N., and Dhillon, N.K., Russ. J. Org. Chem., 2020, vol. 56, p. 113.

https://doi.org/10.1134/S1070428020010182

14. Shupenyuk, V.I., Taras, T.N., Sabadakh, O.P., Luchkevich, E.R., and Kornii, Y., Fr.-Ukr. J. Chem., 2020, vol. 8, p. 58. https://doi.org/10.17721/fujcV8I1P58-65

15. Baqi, Y. and Muller, C.E., Tetrahedron Lett., 2012, vol. 53, p. 6739. https://doi.org/10.1016/j.tetlet.2012.09.011

16. Glushkova, M.A., Popkov, S.V., and Burdeinyi, M.L., Russ. J. Org. Chem., 2020, vol. 56, p. 390. https://doi.org/10.1134/S1070428020030045

17. Lagunin, A., Ivanov, S., Rudik, A., Filimonov, D., and Poroikov, V., Bioinformatics, 2013, vol. 29, p. 2062. https://doi.org/10.1093/bioinformatics/btt322

18. Szklarczyk, D., Morris, J.H., Cook, H., Kuhn, M., Wyder, S., Simonovic, M., Santos, A., Doncheva, N.T., Roth, A., Bork, P., Jensen, L.J., and von Mering, C., Nucleic Acids Res., 2017, vol. 45, p. D362. https://doi.org/10.1093/nar/gkw937 